\title{
SRA-MSDU: Enhanced A-MSDU frame aggregation with selective retransmission in 802.11n wireless networks
}

\begin{abstract}
The main goal of the IEEE 802.11n standard is to achieve more than $100 \mathrm{Mbps}$ of throughput at the MAC service access point. This high throughput has been achieved via many enhancements in both the physical and MAC layers. One of the MAC enhancements is the frame aggregation in which multiple frames are concatenated into a single large frame before being transmitted. The 802.11n MAC layer defines two types of aggregation, aggregate MAC service data unit (A-MSDU) and aggregate MAC protocol data unit (A-MPDU). The AMPDU outperforms A-MSDU due to its large aggregation size and the subframes retransmission in erroneous channels. However, in error free channels and under the same aggregation size the A-MSDU performs better than the A-MPDU due to its smaller headers. Thus, adding a selective retransmission capability to the A-MSDU would improve the system performance. In this paper, we have proposed an MSDU frame aggregation scheme that enables selective retransmission at the MSDU level without altering the original MAC header. In this proposed scheme an implicit sequence control mechanism has been introduced in order to keep the frames in sequence and preserve their correct order at the receiver side. The results show that the proposed scheme improves the system performance in terms of throughput and delay even under highly erroneous channels.
\end{abstract}

Keyword: Frame aggregation; Selective retransmission; SRA-MSDU; A-MSDU; Next generation networks; 802.11n 